\title{
Research on Feasibility of Large-scale New Energy Islanding Detection Scheme Based on Impedance Measurement
}

\author{
Wang Miao \\ Graduate Faculty, China Electric Power Research Institute, Beijing, China
}

\section{Email address:}

wangmiaotj@126.com

\section{To cite this article:}

Wang Miao. Research on Feasibility of Large-scale New Energy Islanding Detection Scheme Based on Impedance Measurement. Science Discovery. Vol. 5, No. 1, 2017, pp. 69-76. doi: 10.11648/j.sd.20170501.21

Received: March 12, 2017; Accepted: April 17, 2017; Published: April 20, 2017

\begin{abstract}
A lot of research has been done on islanding detection of photovoltaic(PV) power system. In these schemes, islanding can be detected by schemes based on convertor control. The range of these research is islanding occurs in a single power station or distributed generation system. However, experiments proof that islanding also occurs in large-scale wind farms. Classical islanding detection schemes such as active frequency drift scheme or reactive power disturbance scheme may not suitable to this situation due to the different parameter when islanding occurs in large-scale new energy power stations. Meanwhile, islanding detection scheme based on system impedance detecion is an ideal scheme to detect islanding in large-scale new energy power stations. However, reaearch on islanding detection scheme based on system impedance only compute the impedance of the outlet end of the convertor, in other words, in a single power station and overlook the problems when the scheme is applied to large-scale new energy power stations, such as power quality problem and signal attenuation problem and so on. This artical will analyze the feasibility of using system impedance to detect islanding in large-scale new energy power stations.
\end{abstract}

Keywords: Islanding Detection, System Impedance, New Energy Power Station

\section{大规模新能源孤岛阻抗测量方法适用性研究}

\section{王沝}

研究生部, 中国电力科学研究院, 北京, 中国

\section{邮箱}

wangmiaotj@126.com

摘要：传统的孤岛检测研究大多关注的是光伏发电系统的孤岛检测, 通过光伏逆变器单机控制来实现, 而且研究针对 的范围是单一电站发生孤岛或者分布式电源及负载发生孤岛运行的情况。实验证明, 不仅小型光伏发电系统可能产生 孤岛现象, 大规模新能源电站也可能出现孤岛运行情况。此时经典的主动偏移法及功率扰动孤岛检测方法由于不同电 站逆变器或者风电机组主动孤岛参数不同可能造成孤岛检测失败。而基于阻抗测量的孤岛检测方法比较容易满足大规 模多电站的孤岛检测要求。既有的基于阻抗测量的研究都是对单逆变器出口侧进行阻抗测量, 并未对其在大规模新能 源电站应用而产生的电能质量恶化问题和因变压器及输电线路对扰动信号的滤波作用造成扰动信号衰减等问题进行分 析。针对上述问题本文将对两种经典的系统阻抗测量方法在大规模新能源电站中的适用性进行仿真分析。 


\section{1. 引言}

根据中国国家能源局的数据, 2016年中国全年新增风 电装机1930万千瓦, 总并网装机容量达1.49亿千瓦, 为总 发电装机容量 $9 \%$, 全年风电发电量 2410 亿千瓦时, 为总发 电量的 $4 \%$; 同时, 2016 年光伏新增装机容量 3454 万千瓦, 总装机容量 7742 万千瓦, 为总发电装机容量 $4.67 \%$, 全年 光伏发电量 662 亿千瓦时, 为总发电量的 $1 \%$ 。中国新能源 虽然装机容量大发展速度快, 但集中式分布特点明显, $80 \%$ 以上集中在消纳能力有限的东北西北华北地区。近年来, 学者对新能源电站大规模接入的研究工作主要集中在对 电力系统潮流分布、稳定性、调峰调频的影响等方面, 很 少关注大规模新能源电站的孤岛问题。

孤岛现象, 是指包含负荷和电源的部分电网, 从主网 脱离后继续孤立运行的状态。既有对孤岛问题的研究多集 中在对光伏逆变器控制的研究, 即改变光伏逆变器的电流 环或功率外环, 并对逆变器单体出口侧电压电流值的特性 进行分析, 以对孤岛进行判别。方法主要分为被动式孤岛 检测方法 [1-3]（过/欠电压法、过/欠频率法、相位跳变
检测法和电压谐波检测法等) 与主动式孤岛检测方法 [4-5] (主动偏移法、功率扰动方法等)。研究表明被动式孤岛 检测方法存在检测盲区大的缺点, 而主动式孤岛检测方法 在多机并联运行的情况下具有可能相互干扰导致孤岛检 测失败的缺点。文献 [6-7]研究在多逆变器并联系统不同 孤岛检测方法配合的特性, 主要对移频和移相两类主动孤 岛检测方法相互联系及影响进行了分析, 为在大规模电站 中不同电站采用不同的孤岛检测方法时可能造成孤岛检 测失败提供了理论依据。同时, 实验也证明大规模新能源 电站孤岛运行的存在。

\section{2. 多电站的孤岛现象}

研究和实际案例表明, 大规模新能源电站也会出现孤 岛运行现象。2012年中国电力科学研究院开展 $330 \mathrm{kV}$ 线路 短路试验, 在试验过程中发现大量风电场发生孤岛运行现 象。实验中发生孤岛风电场如图1所示。

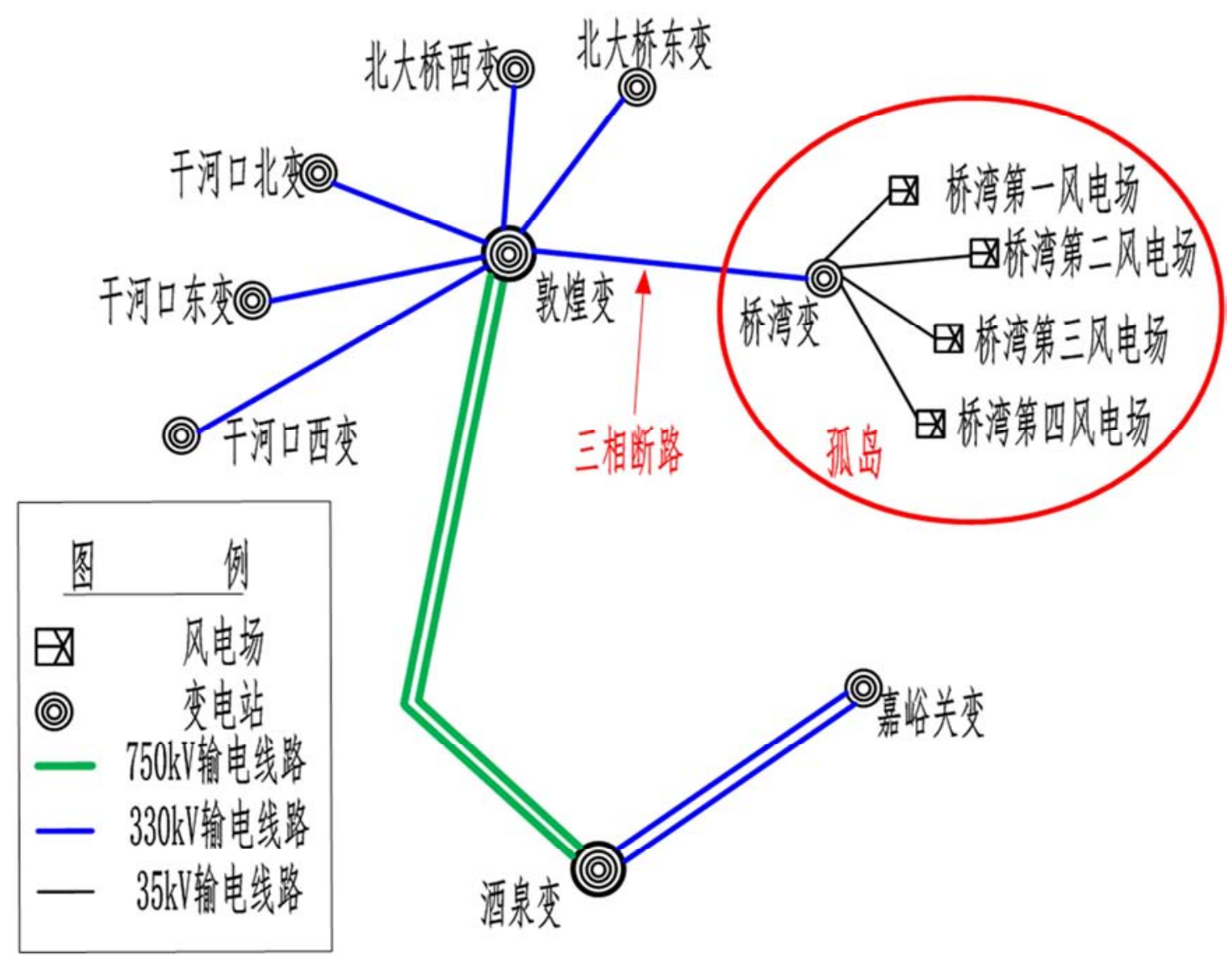

图1 产生孤岛风电场。

实验中, 人为在桥湾变 $330 \mathrm{kV}$ 出线侧制造 $\mathrm{C}$ 相接地故障, $0.7 \mathrm{~s}$ 后 $330 \mathrm{kV}$ 断路器重合闸失败, $330 \mathrm{kV}$ 母线电压降为 0 后, 桥湾一至四号风电场 $35 \mathrm{kV}$ 母线仍有电压存在。图 2 为桥湾 一至四号风电场 $35 \mathrm{kV}$ 母线A相电压波形。孤岛产生后, 四 座风电场 $35 \mathrm{kV}$ 母线都有电压存在, 虽略有波动, 但在 $22 \mathrm{~s}$
时间内基本保持在 $1 \mathrm{pu}$ 。 。这将对运维人员安全及设备安全 造成极大的隐患。

所以研究适用于多风电场或多光伏电站并联运行的 孤岛保护方法具有重要意义。而传统依靠移频或移相的孤 岛检测方法存在互相干扰而导致检测失败的可能性，基于 系统阻抗检测的方法较好的克服了相互干扰的问题。 


\begin{tabular}{|lll|}
\hline Ua_1 & Ua_2 & Ua_3 \\
\hline
\end{tabular}

$\mathrm{U} / \mathrm{Ur} \_\mathrm{p}$

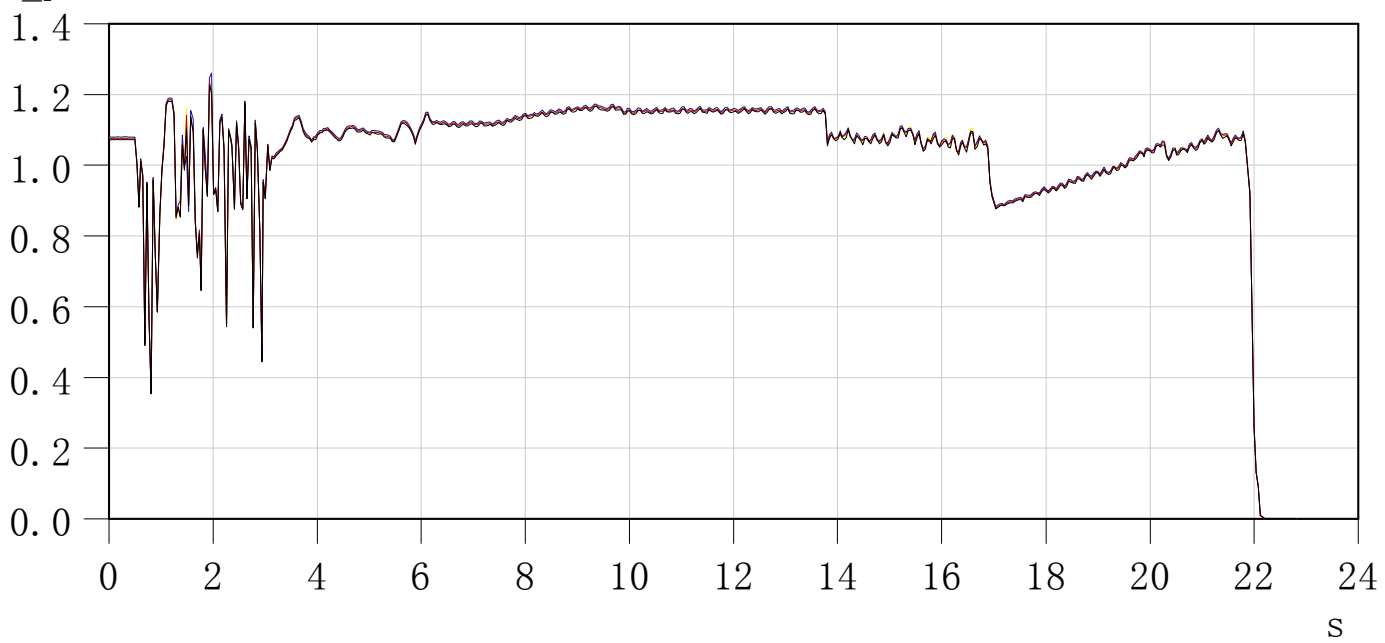

图2 孤岛产生后桥湾一至四号风电场 $35 \mathrm{kV}$ 母线电压。

\section{3．基于阻抗测量孤岛检测方法在大规模新能源孤岛中的适用性分析}

\section{1. 大规模新能源集中并网系统}

\subsection{1. 等效电路模型}

传统针对并网逆变器的电网阻抗测量技术可分为被动测量方式与主动测量方式两种 [8]。被动检测方式精度偏低, 本文主要研究主动测量方式在大规模新能源并网情况下孤岛检测适用性。

$\mathrm{N}$ 座新能源电站三相等效电路如图3所示。其中, 1 至 $\mathrm{N}$ 为电站序号, $Z_{p}$ 为发电系统等效阻抗, $Z_{T}$ 为电站升压变等效 阻抗, $Z_{\text {line }}$ 为电站线路阻抗, $Z_{\text {load }}$ 为电站站内负载等效阻抗, $\mathrm{S}$ 为断路器, 电网阻抗等效为电感 $L_{\text {grid }}$ 。

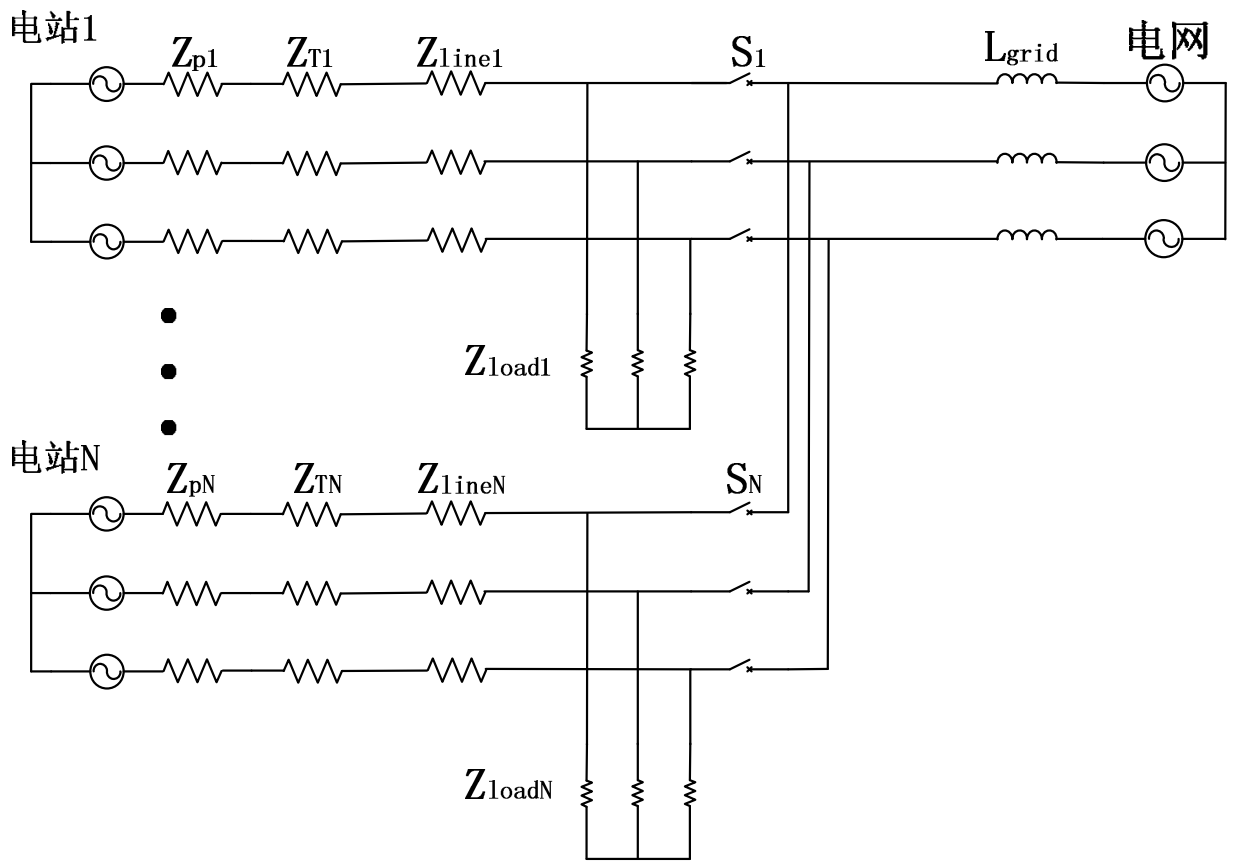

图3 大规模新能源电站电路图。

在三相对称系统中， N个新能源电站并网单相等效电路图如图4所示。 


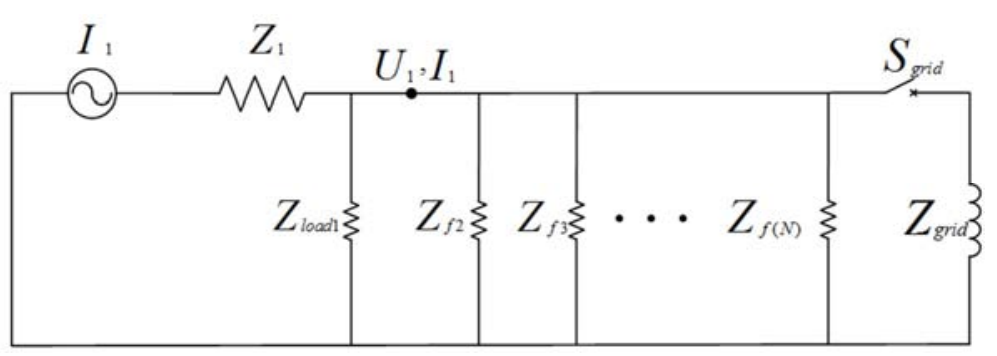

图4 单相等效电路。

将电站 1 等效为电流源 $I_{1}$, 电站 1 内线路及变压器阻抗 等效为 $Z_{1}$, 负载等效为 $Z_{l o a d 1}$, 忽略 $35 \mathrm{kV}$ 线路阻抗, 2 至 $\mathrm{N}$ 号电站阻抗等效为 $Z_{2} 、 Z_{3} \ldots Z_{(N)}$, 电网阻抗等效为 $Z_{\text {grid }}$ 。 序号为 1 的电站并网点电压记为 $U_{1}$, 电流记为 $I_{1}$, 电压电流 不同频率下的分量分别记作 $U_{(j n \omega)} 、 I_{(j n \omega)}$, 可通过对 $U_{1}, I_{1}$ 进行傅里叶分析得到。

$$
Z_{(j n \omega)}=\frac{U_{1(j n \omega)}}{I_{1(j n \omega)}}=\frac{1}{\frac{1}{Z_{2(j n \omega)}}+\cdots+\frac{1}{Z_{N(j n \omega)}}+\frac{1}{Z_{\text {grid }(j n \omega)}}}
$$

在序号为 1 的风电场并网点测量电网侧等效阻抗等于 序号2至N的风电场等效阻抗与电网阻抗的并联阻抗。正常 情况下电网阻抗值 $Z_{\text {grid }}$ 远小于风电场等效阻抗值。孤岛产 生后, 即 $Z_{\text {grid }}$ 支路断开之后, 阻抗幅值明显增加, 且其相 角会由大电网电压决定转变为由孤岛系统阻抗特征决定。
所以可以将阻抗的幅值或相角的变化作为孤岛检测的依 据。基于系统阻抗测量的孤岛检测方法原理比较简单, 对 其研究多集中在阻抗测量的方法上。

\subsection{2. 仿真模型}

为研究阻抗测量方法在大规模新能源电站孤岛检测 中的有效性, 首先在MATlab/simulink环境下搭建大规模 直驱永磁风机电站模型。电站由 $1.5 \mathrm{MW}$ 直驱永磁风机模块、 变压器模块、输电线路模块、RLC并联负载模块、电网模 块组成。模型主接线图如图5所示。仿真过程中断路器 $\mathrm{S}$ 在 $1 \mathrm{~s}$ 时刻断开, 6座风电场发生孤岛, 孤岛持续时间 $1 \mathrm{~s}$, 仿真时长 $2 \mathrm{~s}$, 在测量点 $1 、 2$ 对 $\mathrm{A}$ 相电压电流进行测量。6 座电站风电机组容量如表1所示。

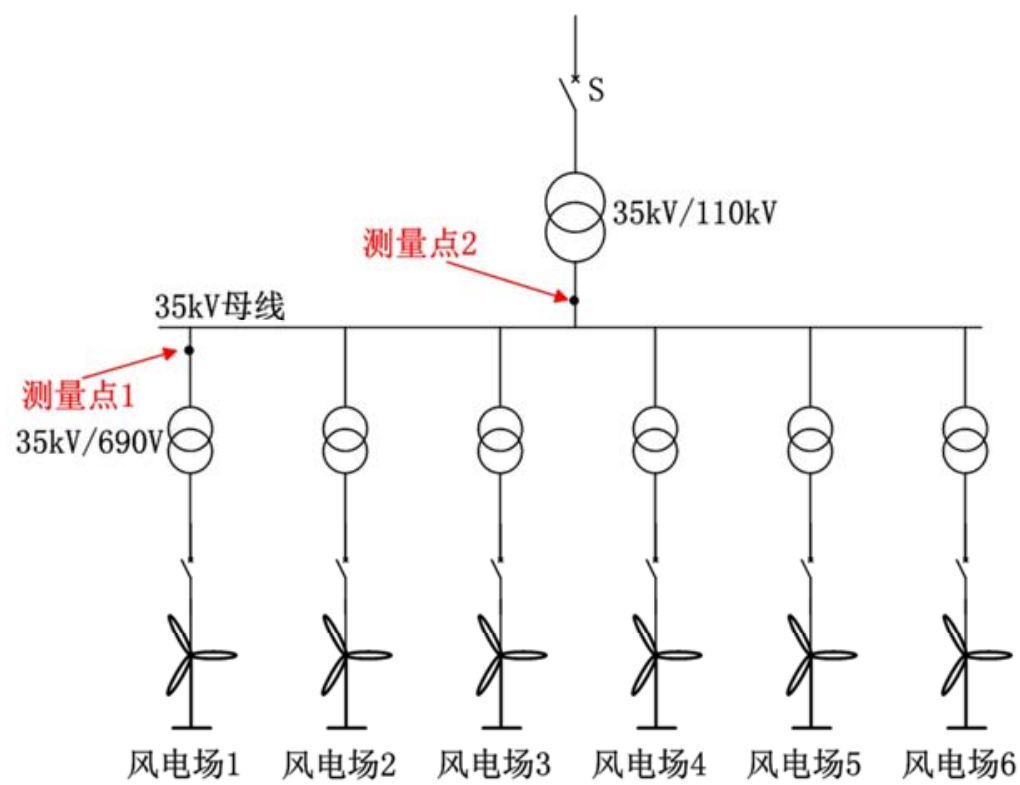

图5 仿真模型主接线图。

表1 仿真模型电站风机容量统计。

\begin{tabular}{llll}
\hline \multirow{2}{*}{ 风电场序号 } & \multicolumn{3}{l}{ 风电场模型风机数量及容量统计 } \\
\cline { 2 - 4 } & 单机容量 $(M W)$ & 风机数量 & 总容量 \\
\hline 1 & 1.5 & 24 & 36 \\
2 & 1.5 & 28 & 42 \\
3 & 1.5 & 24 & 36 \\
4 & 1.5 & 28 & 42 \\
5 & 1.5 & 28 & 42 \\
6 & 1.5 & 48 & 72 \\
7 & 1.5 & 58 & 87 \\
\hline
\end{tabular}

\section{2. 阻抗测量方法}

主动式阻抗测量方法主要可以分为单次谐波注入 (注 入非特征谐波）及复合谐波注入两种方式。这两种方法应 用于大规模电站的孤岛检测中时可能存在如下问题: 1) 在大规模新能源电站孤岛检测时需要以电站为单位, 即在 电站并网点进行电压电流测量并进行快速傅里叶变换并 进行阻抗计算, 而在逆变器侧进行注入的谐波可能由于变 压器和线路的电感电容作用产生衰减, 造成电站出口端电 
压电流的注入频率分量值较小, 造成阻抗计算准确性降低; 2) 大规模新能源电站内大量逆变器同时注入电流谐波可 能造成电网谐波含量升高, 造成电网电能质量恶化。针对 上述问题及阻抗计算精度的影响因素, 本节重点分析单次 谐波扰动注入及复合谐波扰动注入两种方式在大规模新 能源并网环境下的适用性。

\subsection{1. 单次谐波扰动法}

利用注入单次谐波测量电网阻抗是比较直观的方法, 利用在逆变器电流环注入谐波电流作为激励, 测量并网点 电压电流在该频率下的分量, 并利用 $Z_{(j n \omega)}=\frac{U_{(j n \omega)}}{I_{(j n \omega)}}$ 计算该 频率下的系统阻抗值。电流控制框图如图6所示。其中 $G_{c(s)}$ 为调节器、 $K_{P W M}$ 为逆变器等效增益。

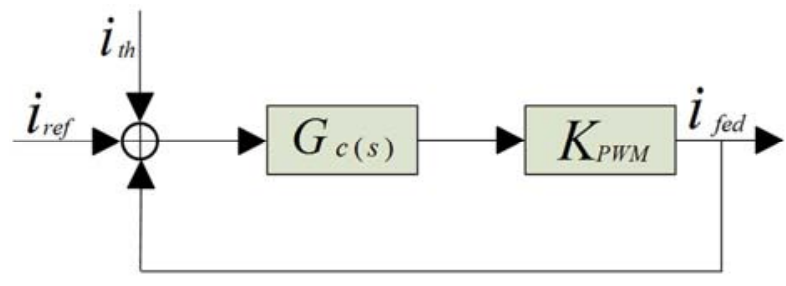

图6 谐波注入电流控制框图。

分别仿真在不同谐波注入幅值和不同谐波注入风机 数量条件下 $35 \mathrm{kV}$ 母线电流谐波含量。参考单逆变器阻抗测 量方法文献 [9-13], 选取注入谐波频率分别为 $75 \mathrm{~Hz}$ 和 $500 \mathrm{~Hz}$ 。下面分析所有风电机组全部施加谐波扰动, 分别 注入 $75 \mathrm{~Hz}$ 及 $500 \mathrm{~Hz}$ 谐波电流时, 注入扰动电流幅值对测量 点2处电流总谐波畸变率（THD）的影响。

表 2 注入 $75 \mathrm{~Hz}$ 谐波 $35 \mathrm{kV}$ 母线A相电流THD。

\begin{tabular}{lll}
\hline \multirow{2}{*}{$\boldsymbol{I}_{\boldsymbol{t} \boldsymbol{h}} / \boldsymbol{I}_{\boldsymbol{r e f}}$} & $35 \mathrm{kV}$ 母线A相电流THD \\
\cline { 2 - 3 } & 孤岛前 & 孤岛后 \\
\hline $5 \%$ & $0.55 \%$ & $0.97 \%$ \\
$7.5 \%$ & $0.55 \%$ & $0.97 \%$ \\
$10 \%$ & $0.55 \%$ & $0.97 \%$ \\
\hline
\end{tabular}

表3 注入 $500 \mathrm{~Hz}$ 谐波 $35 \mathrm{kV}$ 母线A相电流THD。

\begin{tabular}{lll}
\hline \multirow{2}{*}{$\boldsymbol{I}_{\boldsymbol{t} \boldsymbol{h}} / \boldsymbol{I}_{\boldsymbol{r} \boldsymbol{f}}$} & \multicolumn{3}{l}{$35 \mathrm{kV}$ 母线A相电流THD } \\
\cline { 2 - 3 } & 孤岛前 & 孤岛后 \\
\hline $5 \%$ & $2.51 \%$ & $2.45 \%$ \\
$7.5 \%$ & $3.71 \%$ & $3.51 \%$ \\
$10 \%$ & $4.92 \%$ & $4.59 \%$ \\
\hline
\end{tabular}

表 2 及表 3 为全部风电机组注入特征谐波扰动后测量 点 2 处A相电流THD值, 在注入同等幅值条件下, 注入 $75 \mathrm{~Hz}$ 谐波引起的 $35 \mathrm{kV}$ 母线电流THD远小于注入 $500 \mathrm{~Hz}$ 谐波。以电 能质量作为比较标准, 采用注入 $75 \mathrm{~Hz}$ 谐波优于 $500 \mathrm{~Hz}$ 谐波。 并且当 $\frac{{ }^{{ } t h}}{I_{\text {ref }}}=10 \%$ 时, 注入 $500 \mathrm{~Hz}$ 谐波时引起的电流THD值 已超过国家标准GB/T19963-2011《风电场接入电力系统技 术规定》中对风机并网电流谐波含量的要求。因为如果电 压电流谐波分量过小可能会影响阻抗计算精度, 下面分析 在 $\frac{I_{t h}}{I_{\text {ref }}}=0.05$ 与 $\frac{I_{t h}}{I_{\text {ref }}}=0.75$ 条件下测量点 1 处 $75 \mathrm{~Hz}$ 与 $500 \mathrm{~Hz}$ 谐波电压电流分量, 如表4、表5所示。
表4 75Hz电压电流谐波分量。

\begin{tabular}{|c|c|c|}
\hline \multirow{2}{*}{ 电压电流 } & \multicolumn{2}{|l|}{$I_{t h} / I_{r e f}$} \\
\hline & $5 \%$ & $7.5 \%$ \\
\hline $\mathrm{U}_{75 \mathrm{~Hz}}$ （孤岛前） & $5.7 \angle-10.4^{\circ} \mathrm{V}$ & $5.7 \angle-32^{\circ} \mathrm{V}$ \\
\hline $\mathrm{I}_{75 \mathrm{~Hz}}$ (孤岛前） & 2. $17 \angle 169.7^{\circ} \mathrm{A}$ & $2.25 \angle 149.5^{\circ} \mathrm{A}$ \\
\hline $\mathrm{U}_{75 \mathrm{~Hz}}$ （孤岛后） & $556.8 \angle 236.6^{\circ} \mathrm{V}$ & $638.4 \angle 238.5^{\circ} \mathrm{V}$ \\
\hline $\mathrm{I}_{75 \mathrm{~Hz}}$ (孤岛后） & $4.7 \angle 53.5^{\circ} \mathrm{A}$ & 5. $36 \angle 56^{\circ} \mathrm{A}$ \\
\hline
\end{tabular}

表5 500Hz电压电流谐波分量。

\begin{tabular}{lll}
\hline \multirow{2}{*}{ 电压电流 } & \multicolumn{1}{l}{$\boldsymbol{I}_{\boldsymbol{t} \boldsymbol{h}} / \boldsymbol{I}_{\boldsymbol{r e f}}$} & $7.5 \%$ \\
\cline { 2 - 3 } & $5 \%$ & $22.8 \angle-34.4^{\circ} \mathrm{V}$ \\
$\mathrm{U}_{500 \mathrm{~Hz}}$ (孤岛前) & $14.25 \angle-34.1^{\circ} \mathrm{V}$ & $7.68 \angle 139.5^{\circ} \mathrm{A}$ \\
$\mathrm{I}_{500 \mathrm{~Hz}}$ (孤岛前) & $5.13 \angle 140^{\circ} \mathrm{A}$ & $753.6 \angle 261.2^{\circ} \mathrm{V}$ \\
$\mathrm{U}_{500 \mathrm{~Hz}}$ (孤岛后) & $508.8 \angle 260.5^{\circ} \mathrm{V}$ & $6.67 \angle 130.8^{\circ} \mathrm{A}$ \\
$\mathrm{I}_{500 \mathrm{~Hz}}$ (孤岛后) & $4.42 \angle 129.9^{\circ} \mathrm{A}$ & \\
\hline
\end{tabular}

由表4表5可见, 在系统电感电容作用下扰动信号发生 衰减，低压侧注入 $75 \mathrm{~Hz} 、 500 \mathrm{~Hz}$ 谐波含量占基波含量 $5 \%$ 、 $7.5 \%$, 测量点 1 处 $75 \mathrm{~Hz} 、 500 \mathrm{~Hz}$ 谐波含量占基波含量的 $2 \%-4 \%$ 。 由此计算得阻抗值如表6所示。

表6 阻抗计算值。

\begin{tabular}{lll}
\hline \multirow{2}{*}{ 阻抗 } & $\boldsymbol{I}_{\text {th }} / \boldsymbol{I}_{\text {ref }}$ \\
\cline { 2 - 3 } & $5 \%(\Omega)$ & $7.5 \%(\Omega)$ \\
\hline$Z_{75 \mathrm{~Hz}}$ (孤岛前) & $2.627 \angle-180.1^{\circ}$ & $2.53 \angle-181.5^{\circ}$ \\
$Z_{75 \mathrm{~Hz}}$ (孤岛后) & $118.409 \angle 183.1^{\circ}$ & $119.045 \angle 182.5^{\circ}$ \\
$Z_{500 \mathrm{~Hz}}$ (孤岛前) & $2.777 \angle-174.1^{\circ}$ & $2.970 \angle-173.9^{\circ}$ \\
$Z_{500 \mathrm{~Hz}}$ (孤岛后) & $115.056 \angle 130.6^{\circ}$ & $113.04 \angle 130.4^{\circ}$ \\
\hline
\end{tabular}

由表6可见6座电站产生孤岛前后, 测量点1处计算得 到阻抗幅值及相角均发生突变, 虽然扰动信号发生了衰减, 但仍有效且快速的检测到孤岛产生。

若部分风机安装谐波注入装置依然能够准确计算系 统阻抗, 则可以通过该方法减少对电网电能质量的影响。 以下对安装谐波注入模块风机不同数量进行对比。注入 $500 \mathrm{~Hz}$ 谐波扰动, 对安装谐波注入模块风机百分比分别为 $100 \%$ 、60\%进行比较, 测量点 2 处THD及测量点 1 处 $\frac{I_{500 \mathrm{~Hz}}}{I_{50 \mathrm{~Hz}}}$ 及 阻抗计算值如表7所示。

表7 孤岛发生后谐波含量及阻抗计算。

\begin{tabular}{|c|c|c|}
\hline \multirow{2}{*}{ 谐波及阻抗 } & \multicolumn{2}{|c|}{ 安装谐波注入模块风机比例 } \\
\hline & $60 \%$ & $100 \%$ \\
\hline THD（孤岛前） & $1.56 \%$ & $2.51 \%$ \\
\hline$\frac{I_{500 \mathrm{~Hz}}}{I_{50 \mathrm{~Hz}}}$ (孤岛前 $)$ & $1.86 \%$ & $2.46 \%$ \\
\hline THD（孤岛后） & $1.68 \%$ & 2. $45 \%$ \\
\hline$\frac{I_{500 \mathrm{~Hz}}}{I_{50 \mathrm{~Hz}}}($ 孤岛后 $)$ & $1.74 \%$ & $2.21 \%$ \\
\hline $\mathrm{Z}_{500 \mathrm{~Hz}}$ (孤岛前) & $2.477 \angle-174.1^{\circ} \Omega$ & $2.777 \angle-174.1^{\circ} \Omega$ \\
\hline $\mathrm{Z}_{500 \mathrm{~Hz}}$ (孤岛后) & $100.461 \angle 30.1^{\circ} \Omega$ & $115.056 \angle 130.6^{\circ} \Omega$ \\
\hline
\end{tabular}


由表7可见减少安装谐波注入模块风机数量可以有效 减小THD值, 但此方法会导致用以计算阻抗值的电压电流 谐波分量减小，造成阻抗计算值准确度下降。

\subsection{2. 复合谐波扰动法}

单次谐波扰动法对系统在特定频率下的阻抗进行了 测量, 然而由于电网结构与电力设备的复杂多样性, 电网 阻抗与频率之间的关系是非线性的。所以有必要对电网在 不同频率下的阻抗进行测量。对复合谐波的注入, 文献 [14-19]提出在发电机低压侧注入脉冲扰动, 测量电源出 口端线电压从而反映孤岛发生前后系统阻抗的变化, 进而 对分布式电源的孤岛状态进行判定的方法。相对于脉冲信 号, 方波信号是一种更可控并且能在较宽频带对系统阻抗 进行测量的扰动信号。本节将对注入方波扰动信号对系统 阻抗进行测量的方法在大规模电站孤岛检测中的适用性 进行分析。仿真中, 在风机出口侧注入方波扰动。注入方 波信号占空比为 0.33 , 幅值与基波电流幅值之比为 0.03 。 方波信号经快速傅里叶变换 (FFT) 后各次谐波如表8所示。
表8 方波快速傅里叶变换后谐波分量。

\begin{tabular}{ll}
\hline 特征频率/基波频率电流 & 比值 \\
\hline $\mathrm{I}_{10 \mathrm{~Hz}} / \mathrm{I}_{50 \mathrm{~Hz}}$ & $50.23 \%$ \\
$\mathrm{I}_{20 \mathrm{~Hz} \mathrm{z}} / \mathrm{I}_{50 \mathrm{~Hz}}$ & $24.89 \%$ \\
$\mathrm{I}_{25 \mathrm{HH}} / \mathrm{I}_{50 \mathrm{~Hz}}$ & $20.18 \%$ \\
$\mathrm{I}_{350 \mathrm{~Hz}} / \mathrm{I}_{50 \mathrm{~Hz}}$ & $14.16 \%$ \\
$\mathrm{I}_{400 \mathrm{~Hz}} / \mathrm{I}_{50 \mathrm{~Hz}}$ & $12.68 \%$ \\
\hline
\end{tabular}

方波扰动中含有大量 $100 \mathrm{~Hz} 、 200 \mathrm{~Hz} 、 250 \mathrm{~Hz} 、 350 \mathrm{~Hz}$ 及 $400 \mathrm{~Hz}$ 分量, 本文以 5 次与 7 次谐波为例对系统阻抗进行 计算。施加扰动前后测量点 1 处 $35 \mathrm{kV}$ 母线A相电流THD如表 9 所示。施加扰动后 $35 \mathrm{kV}$ 母线A相电流波形如图7所示。

表9 施加扰动前后A相电流THD。

\begin{tabular}{ll}
\hline 工况 & 孤岛前THD \\
\hline 未加扰动 & $0.07 \%$ \\
注入方波扰动 & $0.8 \%$ \\
\hline
\end{tabular}

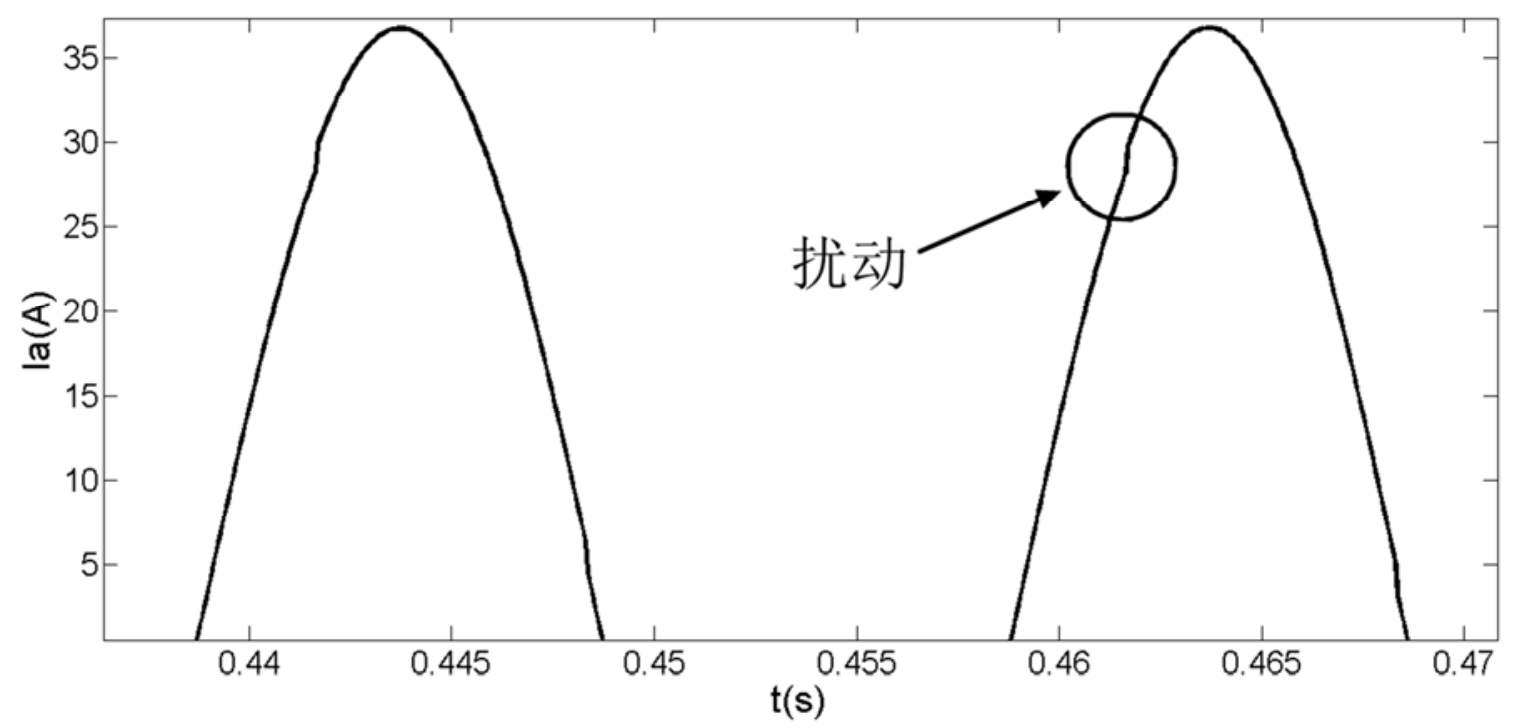

图7 叠加扰动的 $\mathrm{A}$ 相电流波形。

方波扰动信号幅值很小, 所以注入扰动后在孤岛产生 前, 3次、5次及7次谐波电流含量满足GB/T19963-2011对 并网风电机组输出电流谐波的要求。

由于风电场站在正常并网运行时存在含量相对较高 的奇次谐波, 当选取5次与 7 次谐波对系统阻抗进行测量时, 需要考虑系统固有5次和7次背景谐波的影响。首先在不加 方波电流扰动时记录测量点 1 处 $35 \mathrm{kV}$ 母线A相电压电流值, 并分析A相电压电流 $250 \mathrm{~Hz}$ 与 $350 \mathrm{~Hz}$ 谐波含量。然后测量施 加方波电流扰动时 $\mathrm{A}$ 相电压电流 $250 \mathrm{~Hz}$ 与 $350 \mathrm{~Hz}$ 谐波含量。 用施加扰动后的谐波分量减去未加扰动时的谐波分量即 为在方波扰动作为激励下的电压电流分别在 $250 \mathrm{~Hz}$ 与 $350 \mathrm{~Hz}$ 下的响应, 以便对系统在 $250 \mathrm{~Hz}$ 及 $350 \mathrm{~Hz}$ 下的阻抗进 行测量。表 10 及表 11 为注入扰动前后测量点 1 处A相电压电 流 $250 \mathrm{~Hz}$ 及 $350 \mathrm{~Hz}$ 分量孤岛前后幅值与相角。表 12 为由方波 扰动产生的 $A$ 相电压电流变化量, 即用以进行阻抗计算的 电压电流值。
表10 扰动前 $35 \mathrm{kV}$ 母线A相电流 $250 \mathrm{~Hz}$ 及350Hz谐波分量。

\begin{tabular}{lll}
\hline \multirow{2}{*}{ 频率 } & \multicolumn{3}{l}{ 电压电流250Hz、350Hz谐波分量 } \\
\cline { 2 - 3 } & 电压 $(\mathrm{V})$ & 电流 $(\mathrm{A})$ \\
\hline $250 \mathrm{~Hz}$ (孤岛前) & $2.85 \angle 12.9^{\circ}$ & $0.29 \angle 11.7^{\circ}$ \\
$250 \mathrm{~Hz}$ (孤岛后) & $110.22 \angle-57.5^{\circ}$ & $0.14 \angle-77.3^{\circ}$ \\
$350 \mathrm{~Hz}$ (孤岛前) & $2.85 \angle 10.7^{\circ}$ & $0.2 \angle 9.9^{\circ}$ \\
$350 \mathrm{~Hz}$ (孤岛后) & $60.97 \angle-47.4^{\circ}$ & $0.07 \angle-72.2^{\circ}$ \\
\hline
\end{tabular}

表11 扰动后 $35 \mathrm{kV}$ 母线A相电流 $250 \mathrm{~Hz}$ 及350Hz谐波分量。

\begin{tabular}{lll}
\hline \multirow{2}{*}{ 频率 } & \multicolumn{3}{l}{ 电压电流250Hz、350Hz谐波分量 } \\
\cline { 2 - 3 } & 电压 $(\mathrm{V})$ & 电流 $(\mathrm{A})$ \\
\hline $250 \mathrm{~Hz}$ (孤岛前) & $2.85 \angle 48.1^{\circ}$ & $0.16 \angle 22.2^{\circ}$ \\
$250 \mathrm{~Hz}$ (孤岛后) & $101.14 \angle-62.5^{\circ}$ & $0.18 \angle 228.6^{\circ}$ \\
$350 \mathrm{~Hz}$ (孤岛前) & $2.85 \angle 46.9^{\circ}$ & $0.11 \angle 18.9^{\circ}$ \\
$350 \mathrm{~Hz}$ (孤岛后) & $54.1 \angle-52.3^{\circ}$ & $0.11 \angle 218.8^{\circ}$ \\
\hline
\end{tabular}


表 12 方波扰动产生的 $250 \mathrm{~Hz}$ 及 $350 \mathrm{~Hz}$ 电压电流分量。

\begin{tabular}{lll}
\hline \multirow{2}{*}{ 频率 } & \multicolumn{2}{l}{ 电压电流250Hz、350Hz谐波分量 } \\
\cline { 2 - 3 } & 电压 $(\mathrm{V})$ & 电流 $(\mathrm{A})$ \\
\hline $250 \mathrm{~Hz}$ (孤岛前) & $1.724 \angle 120.5^{\circ}$ & $0.138 \angle 179.7^{\circ}$ \\
$250 \mathrm{~Hz}$ (孤岛后) & $12.933 \angle 165.5^{\circ}$ & $0.144 \angle 180^{\circ}$ \\
$350 \mathrm{~Hz}$ (孤岛前) & $1.771 \angle 118.8^{\circ}$ & $0.099 \angle-179.7^{\circ}$ \\
$350 \mathrm{~Hz}$ (孤岛后) & $8.447 \angle 165.8^{\circ}$ & $0.104 \angle-179.5^{\circ}$ \\
\hline
\end{tabular}

由表12所得电压电流值计算得到孤岛前后测量点 1 处 阻抗计算值如表13所示。

表13 阻抗计算值。

\begin{tabular}{ll}
\hline 阻抗计算值 & \\
\hline 频率 & 阻抗 $(\Omega)$ \\
\hline $250 \mathrm{~Hz}$ (孤岛前 $)$ & $12.5 \angle-59.2^{\circ}$ \\
$250 \mathrm{~Hz}$ (孤岛后) & $90.1 \angle-14.5^{\circ}$ \\
$350 \mathrm{~Hz}$ (孤岛前 $)$ & $17.9 \angle-61.5^{\circ}$ \\
$350 \mathrm{~Hz}$ (孤岛后 $)$ & $81.62 \angle-14.7^{\circ}$ \\
\hline
\end{tabular}

由表13可见孤岛产生前后, 测量点1处 $250 \mathrm{~Hz}$ 及 $350 \mathrm{~Hz}$ 阻抗幅值及相角均发生突变, 验证了注入方波电流作为扰 动量以对系统阻抗进行计算的可行性。同样其计算精度受 注入方波的幅值及占空比影响。

\section{4. 结论}

仿真证明，单次谐波扰动注入与复合谐波扰动注入法 均能够有效对大规模新能源电站孤岛产生进行检测, 而注 入谐波的幅值、加入谐波注入模块的发电单元数量、方波 扰动信号占空比等因素都会影响电能质量和阻抗计算精 度: 扰动量越大, 阻抗计算精度越高, 而对电能质量的影 响越大。另外多新能源电站接入电网的不同拓扑结构和新 能源接入地电网强弱以及光伏电站及风电场站的并网与 解列都会造成系统阻抗值不同或者变化, 所以难以确定通 用化的孤岛保护國值, 这些因素对基于阻抗测量的孤岛检 测方法的影响需要我们进一步的研究。

\section{参考文献}

[1] Francesco De M., Marco L., Antonio D. A., et al. "Overview of anti-islanding algorithms for PV systems. Part 1: Passive Methods [C]” . 12th International Power Electronics and Motion Control Conference, 2006. EPE-EPMC, 2006:1878-1883.

[2] Vieira J.C.M., Freitas W., Wilsun X., et al. "Performance of frequency relays for distributed generation protection $[\mathrm{J}]$ ” . 1EEE Transactions on PowerDelivery., vol. 3, pp. 1120-1127, Aug. 1993.

[3] Sung-И J., Kwang-Ho К. , “An islanding detection method for distribution generation using voltage unbalance and total harmonic distortion of current $[\mathrm{J}]$ ”. IEEE
Transactions on PowerDelivery., vol. 19, pp. 745-752, 2004.

[4] Ropp M. E., Begovic M., Rohatgi A. “Analysis and performance assessment of the active frequency drift method of islanding prevention [J]" . IEEE Transaction on Energy Conversion., vol. 3, pp. 810-816, 1999.

[5] Jin Beom J., Hee Jun K, Kang Soon A., et al. “novel method for anti-islanding using reactive power[C]" 27th International Telecommunications Conference, NTELEC' 05, 2005: 101-106.

[6] 刘方锐, 段善旭, 康勇, 张宇. 多机光伏并网逆变器的孤岛 检测技术 $[J]$. 电工技术学报, 2010, 25(1): 167-171。

[7] 刘方锐, 余蜜, 张宇, 段善旭, 康勇. 主动移频法在光伏并 网逆变器并联运行下的孤岛检测极力研究。

[8] 谢少军, 季林, 许津铭. 并网逆变器电网阻抗检测技术综述 [J]. 电网技术，2015, 39(2):320-326。

[9] 胡国珍, 段善旭, 陈昌松, 等. 弱电网下储能电池能量回馈 系统自适应并网控制策略 $[\mathrm{J}]$. 电力系统自动化, 2013, 37 (1) :84-89。

[10] Asiminoaei L, Teodorescu R, Blaabjerg F, et al. A digital controlled PV-inverter with grid impedance estimation for ENS detection [J]. IEEE Transactions on Power Electronics, 2005, 20(6): 1480-1490.

[11] Asiminoaei L, Teodorescu R, Blaabjerg F, et al. Implementation and test of an online embedded grid impedance estimation technique for PV inverters $[\mathrm{J}]$, IEEE Transactions on Industrial Electronics, 2005, $52(4): 1136-1144$.

[12] Ciobotaru M, Teodorescu R, Blaabjerg F. On-line grid impedance estimation based on harmonic injection for grid-connected PV inverter [C], 2007 IEEE International Symposium on Industrial Electronics. Vigo, Spain: IEEE, 2007: 2437-2442.

[13] 汤婷婷, 张兴, 谢东, 等. 基于高频注入阻抗检测的孤岛检 测研究 $[J]$. 电力电子技术, 2013, 47 (3) : 70-72。

[14] 韩耀鹏. 基于可控短路技术的微电网孤岛检测技术研 究. [D]. 山东大学, 2011。

[15] J. Kliber, Wencong Wang, Wilsun Xu. Local Anti-Islanding Protection for Distributed Generators Based on Impedance Measurements. IEEE Electric Power Conference, pp. 1-5, 0ct. 2008.

[16] 卢晓惠. 基于系统阻抗测量的有源孤岛保护技术研究. [D]. 山东大学, 2013。

[17] Roinila T, Vilkko M, Sun J. Broadband methods for online grid impedance measurement [C]. 2013 IEEE Energy Conversion Congress and Exposition. Denver, C0: IEEE, 2013: 3003-3010. 
[18] Martin D, Santi E, Barklay A. Wide bandwidth system identification of AC system impedances by applying pertubations to an existing converter[C]. 2011 IEEE Energy Conversion Congress and Exposition. Phoenix, AZ: IEEE, 2011:2549-2556.
[19] Roinila T, Vilkko M, Sun J. Online grid impedance mearsurement using discrete-interval binary sequence injection[C]. 2013 IEEE 14th Workshop on Control and Modeling for Power Electronics. Salt Lake City, UT: IEEE, 2013:1-8. 